Audiology

Neurotology
Audiol Neurotol 2009;14:361-372

DOI: $\underline{10.1159 / 000241893}$
Received: April 26, 2009

Accepted: May 8, 2009

Published online: November 16, 2009

\title{
Vestibular Function and Vertigo Control after Intratympanic Gentamicin for Ménière's Disease
}

\author{
Kimanh D. Nguyen ${ }^{a}$ Lloyd B. Minor ${ }^{a-c}$ Charles C. Della Santina ${ }^{a, b}$ \\ John P. Carey ${ }^{\mathrm{a}}$ \\ ${ }^{a}$ Department of Otolaryngology - Head and Neck Surgery, Johns Hopkins University School of Medicine, and \\ Departments of ${ }^{b}$ Biomedical Engineering and ${ }^{c}$ Neuroscience, Johns Hopkins University, Baltimore, Md., USA
}

\section{Key Words}

Ménière's disease $\cdot$ Gentamicin $\cdot$ Angular vestibulo-ocular reflex $\cdot$ Caloric unilateral weakness $\cdot$ Vertigo

\begin{abstract}
The aim of this study was to correlate long-term vertigo control with reduction in vestibular function after intratympanic (IT) gentamicin therapy for unilateral Ménière's disease. IT gentamicin injections were given as needed to control vertigo attacks. Vertigo frequency and changes in angular vestibulo-ocular reflex (AVOR) gain (measured using magnetic search coils and manual head thrusts) and caloric weakness were assessed before and after treatment. Better vertigo control after treatment was found with $\geq 60 \%$ reduction in quantitative ipsilateral horizontal semicircular canal AVOR gain from pre-treatment values and/or with caloric unilateral weakness (UW) $>50 \%$. However, no correlations were found between the continuous variables of vertigo control and either gain or gain recovery, nor between gain and UW because of the large variability in vertigo control in subjects with lesser reductions in these measures.
\end{abstract}

Copyright $\odot 2009$ S. Karger AG, Basel

\section{KARGER}

Fax +4161306 1234

E-Mail karger@karger.ch

www.karger.com
(C) 2009 S. Karger AG, Basel

Accessible online at: www.karger.com/aud

\section{Introduction}

Ménière's disease is an inner ear disorder of episodic vertigo, sensorineural hearing loss, aural fullness, and tinnitus [Monsell et al., 1995] that affects approximately 15 out of every 100,000 individuals in the United States [Wladislavosky-Waserman et al., 1984], with similar values of prevalence reported throughout the world [Stahle et al., 1978; Watanabe et al., 1995; Kotimaki et al., 1999; da Costa et al., 2002]. The disabling symptoms of vertigo in Ménière's disease may be treated with intratympanic (IT) injections of the ototoxic aminoglycoside gentamicin [Lange, 1989; Chia et al., 2004; Cohen-Kerem et al., 2004].

Prior investigations have also sought to determine if vestibular testing can predict the degree of vertigo control and provide an endpoint for gentamicin treatment. Hone et al. [2000] found that patients with absent ice water responses were significantly less likely to have recurrent vertigo than those with persistent caloric responses. However, the same group subsequently observed that there was no correlation between these 2 variables when patients were followed for a more extended period of time after treatment [Bodmer et al., 2007]. De Waele et al. [2002] suggested that gentamicin be given until otolith 
endorgan function was ablated. They found that patients who lost the galvanically evoked VEMP (vestibular evoked myogenic potential) response after IT gentamicin treatment - and who therefore presumably had damage beyond the hair cells to include the afferent neurons of the otolith organs - did not experience recurrence of vertigo. Lin et al. [2005] reported that partial vestibular ablation appeared to be beneficial, by showing that patients who did not suffer vertigo recurrence had significantly greater decreases in ipsilateral horizontal canal angular vestibulo-ocular reflex (AVOR) gain 1 year after treatment than those who had recurrent vertigo.

While these past studies have focused on the effectiveness of various vestibular tests in predicting long-term vertigo control, none have specifically addressed the issue of recurrence of vertigo and how it may be correlated with any recovery of vestibular function. Vertigo recurrence has been reported to occur in $0-71 \%$ of patients treated with IT gentamicin under various protocols [Cohen-Kerem et al., 2004]. Previous research from our laboratory found that patients required an average of 2.5 injections to achieve vertigo control. We applied a survival analysis to describe the time course of recurrence of vertigo after IT gentamicin treatment, and in that study $46 \%$ of patients had recurrent vertigo that required additional injections of gentamicin [Nguyen et al., 2009]. The goal of the present study was to determine which, if any, measures of vestibular function correlate with recurrence of vertigo in Ménière's disease after IT gentamicin treatment. We examined both long-term vertigo control and the recurrence of vertigo, as well as long-term vestibular function as measured by the AVOR in response to the rapid rotary head thrust test (HTT) and caloric tests.

\section{Patients and Methods}

\section{Patients Included in the Analysis}

Forty-eight patients with unilateral Ménière's disease as defined by the 1995 criteria of the American Academy of Otolaryngology - Head and Neck Surgery were treated with IT gentamicin for vertigo refractory to medical treatment (salt-restricted diet, diuretics, vestibular suppressant and anti-emetic medications) [Monsell et al., 1995]. The patients were drawn from a comprehensive database of 97 patients who were treated at our institution with IT gentamicin between 21 July 2000 and 26 May 2006. At the time of the final testing reported here, follow-up ranged from 5.7 months to 8.8 years for these patients (median 3.7 years). All patients gave informed consent for the AVOR recordings through a protocol approved by the Institutional Review Board at the Johns Hopkins University School of Medicine, the institution at which treatment and testing were performed. Pre-treatment and post- treatment AVOR gain data for 18 of these patients have previously been included in an earlier publication [Lin et al., 2005].

\section{Protocol for Gentamicin Treatments}

Gentamicin was administered as we have previously described [Minor, 1999]. The mid-posterior aspect of the tympanic membrane was anesthetized and punctured, and the middle ear was filled with a buffered gentamicin solution $(26.7 \mathrm{mg} / \mathrm{ml}$ gentamicin, $0.4 \mathrm{ml}$ typically injected). Patients remained supine with the head angled slightly head down and turned to the contralateral side for 30 min to continually bathe the round window with gentamicin solution. The solution was then aspirated from the external canal. Each patient received only 1 injection during this first round.

Subsequent rounds of treatment were similarly given if patients had recurrent vertigo. At least 6 weeks elapsed between the first injection of a given treatment round and the first injection of a subsequent treatment round. For the majority of patients (46 of 48 ), each round consisted of a single injection. Patients were asked to complete caloric and quantitative head thrust testing before and after each treatment round.

\section{Caloric Tests}

Caloric tests were performed using a temperature switch irrigation technique for 30.5 and $43.5^{\circ} \mathrm{C}$ [Proctor et al., 1975]. Horizontal eye movements were recorded with electro-oculography, and maximum velocity of the slow-phase component of nystagmus was analyzed for unilateral weakness (UW) and directional preponderance as determined by conventional formulas [Jongkees et al., 1962]. An ice water caloric test was performed when there was no response to warm or cold irrigation of the affected ear (100\% asymmetry). If nystagmus was noted in response to the ice water test, the patient was turned from supine to prone to see if the nystagmus reversed direction, as expected based upon a convective mechanism [Paige, 1985; Minor and Goldberg, 1990].

\section{Qualitative (Clinical) HTT}

Prior to quantitative testing, a qualitative ('bedside') HTT of the AVOR was performed. The patient was seated and asked to maintain gaze on the examiner's nose. The examiner sat in front of the patient and grasped the patient's head over the temporoparietal areas. The examiner rapidly rotated the head by $10-20^{\circ}$ in the horizontal plane, either to the left to excite the left horizontal canal or to the right to excite the right horizontal canal. The patient could not predict the direction of the rotation. The integrity of the AVOR in each direction was qualitatively graded as 'normal' if the eyes remained stable in space on all trials. It was graded as 'mildly diminished' if a refixation saccade could be detected on some but not all trials, 'diminished' if a refixation saccade could be detected on all trials, and 'markedly diminished' if all trials demonstrated a refixation saccade with a long latency or a multistep refixation saccade. The same experienced examiner performed all of these assessments (J.P.C.).

\section{Quantitative HTT and Gain Calculation}

Generally, pre-treatment HTTs were performed on the same day as treatment, and post-treatment tests were performed 6 weeks following a round of treatment.

The patient, seated with the head centered in the magnetic field, was instructed to gaze at a light-emitting diode located 124 
$\mathrm{cm}$ directly forward at eye level. The examiner stood behind the patient and grasped the head over the temporoparietal areas. The head was kept stationary in a comfortable 'upright' position prior to each head thrust. This position placed the Frankfort line (inferior orbital rim to superior external auditory canal) $7 \pm 7^{\circ}$ noseup from the earth-horizontal plane. From this position, the examiner rapidly rotated the head by $10-20^{\circ}$ in the horizontal plane. The head was turned to the left to excite the left horizontal canal or to the right to excite the right horizontal canal. The patient could not predict the direction of the rotation. Approximately 10 20 head thrusts in either direction were performed.

The instrumentation and technique for recording eye and head movements during these head thrusts with magnetic search coils have been described in detail elsewhere [Straumann et al., 1995]. Our protocol for measuring AVOR reflexes elicited by manual head thrusts and for calculating gains has also previously been described [Carey et al., 2002]. In brief, monocular or binocular eye movements were recorded in 3 dimensions at $500-\mathrm{Hz}$ sampling rates using magnetic search coils embedded in scleral contact lenses (Skalar, Delft, The Netherlands). Head movements were recorded with magnetic search coils attached to a Plexiglas plate coated with hardened dental impression compound and molded to the patient's dental occlusion. Eye and head positions in 3 dimensions were expressed as rotation vectors and used to derive the angular velocities of the eye and head. We discarded data from head thrusts during which the eye responses included blinks, saccades, or eye movements that commenced before the onset of head movement. The starting point of the head thrust was taken when the measured head velocity exceeded the mean velocity at rest, which was close to $0 \%$, by $8 \times$ standard deviation + $2 \%$ [Tabak et al., 1997]. For each head thrust, we calculated AVOR gain at each sampling point as the ratio of horizontal eye velocity/ horizontal head velocity. As a representative 'best value' of gain for each head thrust, we took the highest gain value that occurred in a $30-\mathrm{ms}$ period prior to peak head velocity. Later times are avoided because of the possible intrusion of non-vestibular eye movements, particularly the refixation saccades that may compensate for a deficient AVOR in the early portion of the head thrust [Tian et al., 2000]. The rotational data were analyzed using a program that we developed for the Labview software platform (National Instruments, Austin, Tex., USA). Means and SD were calculated for 5-20 trials exciting each of the horizontal canals.

\section{Data Analysis}

Vertigo Rates

At each visit, patients were asked to provide the number of vertigo attacks that had occurred since the last visit. A monthly rate of vertigo attacks was calculated based on this number. It should be noted that monthly vertigo rates were, in some cases, calculated based on a small number of attacks, sometimes even one. This was necessary because of the 'titration' nature of our approach to gentamicin treatment, in which patients were given repeated injections of IT gentamicin when the patient felt that they were needed to control vertigo. Paired, 2-tailed t-tests were used to compare pre- and post-treatment rates of vertigo occurrence.

\section{Gain Recovery}

Percent recovery of ipsilateral horizontal canal AVOR gain was calculated according to the following equation, where $G$ rep- resents ipsilateral horizontal canal gain, $t_{1}$ is the pre-treatment time point, $t_{2}$ is an early post-treatment time point, and $t_{3}$ is a late post-treatment time point.

Equation for calculation of percentage recovery, where $t_{2} \mathrm{Oc}-$ curs at least 2 weeks following the first IT gentamicin injection:

Percentage recovery $(\%)=100 \times\left[G\left(t_{3}\right)-G\left(t_{2}\right)\right] / G\left(t_{1}\right)$

Statistical Analysis

Analyses of significance levels were performed using Student's t test, $\chi^{2}$ analysis with Yates' correction, Fisher's exact test, or Mann-Whitney $U$ analysis, where appropriate. Linear regression and correlation coefficients were used to determine associations between continuously plotted values. Student's t tests and correlation coefficient analysis were performed using Microsoft Excel 2003 (Microsoft, Redmond, Wash., USA). Binary logistic regression analyses and Mann-Whitney $U$ analyses were performed using Minitab Versions 11 and 15 (Minitab, State College, Pa., USA); $\chi^{2}$ analysis with Yates' correction and Fisher's exact test were calculated using GraphPad (http://graphpad.com/quickcalcs/contingencyl.cfm).

\section{Results}

\section{Section I - Vertigo Recurrence}

Twenty-four patients out of 48 (50\%) had sufficient vertigo control following the first gentamicin injection such that no additional IT gentamicin injections were needed. The remaining 24 patients (50\%) had vertigo recurrence necessitating additional injections. For these patients, the average time to recurrence of vertigo was 7.4 \pm 9.6 months (range 13 days to 46.6 months, median 4.7 months). Patients were followed for a mean of $37 \pm 22$ months (range 6 months to 6.2 years) after their first IT gentamicin injection.

Figure 1 depicts the rate of vertigo attacks per month for each of the 24 patients with unilateral Ménière's disease who required more than one IT gentamicin injection for control of their vertigo. Pre-treatment vertigo rates before the first IT gentamicin injection are plotted at time 0 , and post-treatment vertigo rates at all available followup points are plotted at the appropriate time points. The color scale indicates the frequency of vertigo attacks estimated by the patient at each visit. White-bordered boxes filled with the darkest blue color indicate visits in which the patient confirmed that he or she had complete vertigo control. Follow-up data are available for the majority of patients, and, in particular, there are more follow-up data for patients who started out with more frequent vertigo attacks (right side of plot).

The majority of patients had, by the time of analysis, complete vertigo control as indicated by the zero-vertigo (white-bordered, dark blue-filled) boxes being the top- 


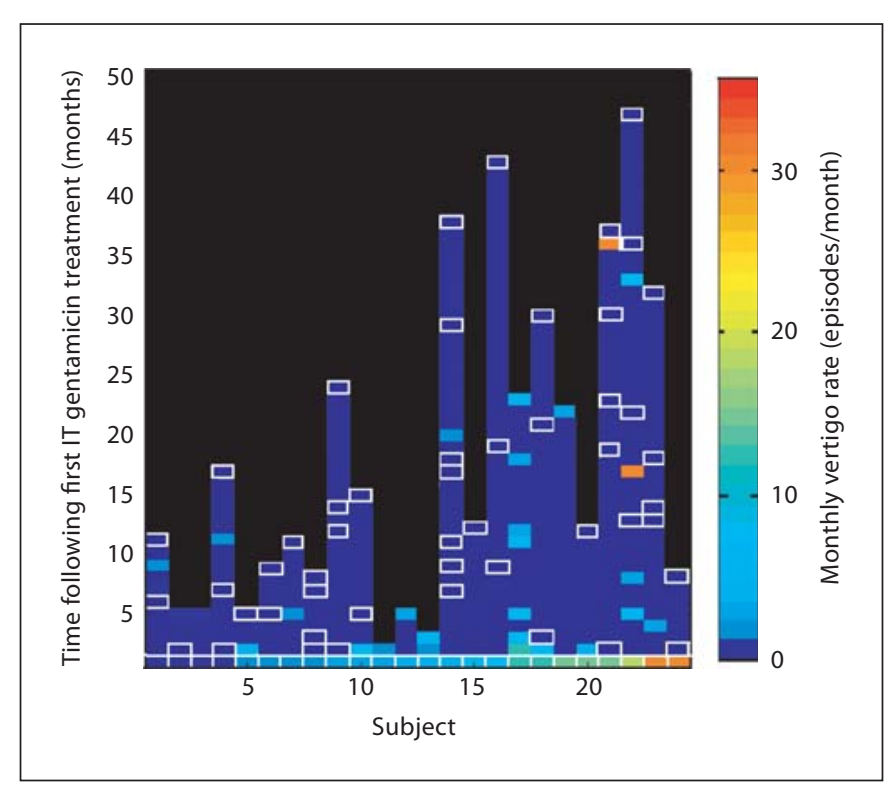

Fig. 1. Vertigo rates after the first IT gentamicin treatment. Pretreatment vertigo rates are shown at time 0 for each of 24 patients, and post-treatment rates are shown at later time points. Whitebordered boxes indicate reported vertigo rates of 0 . For boxes indicating a non-zero frequency of vertigo, the duration of time with this frequency of vertigo attacks and the actual number of attacks are not depicted; rather, the rate is expressed as a point measure at the time of retreatment.

most data points. The graph demonstrates that patients who had low vertigo rates at the initiation of treatment tended to have any recurrences early. In fact, recurrences were encountered mostly within 10 months for the half of these patients to the left of the graph. In contrast, patients who had severer vertigo at enrollment tended to have more recurrences, and these recurrences were widely distributed over time, even as late as 73 months after the initial injection.

\section{Section II - Clinical (Qualitative) HTT}

Data on the qualitative clinical HTT results after the first IT gentamicin treatment were available for 33 patients. Data on pre-treatment clinical HTT results were available for 23 of these 33 patients: 18 had normal HTT pre-treatment results, 4 had slightly diminished HTT results, and 1 had a diminished HTT response.

After IT gentamicin treatment, out of 33 individuals, 15 (45.5\%) had normal or mildly diminished HTT results on the affected side, and the remaining 18 (54.5\%) had diminished or markedly diminished HTT results on that side. Ten out of 15 patients (66.7\%) with normal or mild-
Table 1. Rates of vertigo before and after the first round of gentamicin treatment

\begin{tabular}{lllll}
\hline Group & $\begin{array}{l}\text { Original ipsilateral } \\
\text { HC AVOR } \\
\text { gain retention, \% }\end{array}$ & $\begin{array}{l}\text { Sample } \\
\text { size }\end{array}$ & $\begin{array}{l}\text { Pre-treatment } \\
\text { vertigo rate } \\
\text { episodes/month }\end{array}$ & $\begin{array}{l}\text { Post-treatment } \\
\text { vertigo rate } \\
\text { episodes/month }\end{array}$ \\
\hline 1 & $20-39.9$ & 11 & $13.1 \pm 21.1$ & $0.27 \pm 0.47$ \\
2 & $\geq 40$ & 37 & $11.9 \pm 15.9$ & $1.8 \pm 4.4$ \\
\hline
\end{tabular}

$\mathrm{HC}=$ Horizontal canal.

ly diminished HTT results had vertigo recurrence, compared with only 7 out of 18 patients (38.9\%) with diminished or markedly diminished results ( $\chi^{2}$ test with Yates' correction: $\chi^{2}=1.538, p=0.2149$; power $\left.=47.8 \%\right)$. Thus, qualitative results of the HTT after the first IT gentamicin treatment were not significantly different between the patients who went on to have recurrent vertigo and those who did not.

\section{Section III - Quantitative HTT}

Early Gain Changes after IT Gentamicin Treatment, Vertigo Control, and Vertigo Recurrence

Table 1 shows the pre- and post-treatment vertigo rates corresponding to the first IT gentamicin injection in 48 patients, divided into 2 groups: group 1 included those who retained $<40 \%$ of their original AVOR gain; group 2 included those who retained $\geq 40 \%$ of original AVOR gain. Both groups experienced pronounced decreases in vertigo rate following IT gentamicin treatment, although only the drop for group 2 reached statistical significance (group 1, $\mathrm{p}=0.0721$; group $2, \mathrm{p}=0.000258$ ). However, the difference in post-treatment vertigo rates between the groups was statistically significant ( 0.27 vs. 1.8 episodes/month, $\mathrm{p}=0.045$, 2 -tailed $\mathrm{t}$ test). Thus, vertigo rates on average dropped after the first round of IT gentamicin treatment regardless of the amount of AVOR gain reduction, but an AVOR gain reduction to a value $<40 \%$ of the pre-treatment value was more predictive of lower post-treatment vertigo rates than was a less severe reduction in AVOR gain.

Despite the significantly greater reduction in posttreatment vertigo rate associated with the greater reduction in AVOR gain in group 2, some of these patients (as well as some in group 1) nevertheless considered their recurrent vertigo troublesome enough to seek additional IT gentamicin injections. In this regard, it is important to note that there was not a significant difference in the probability of some vertigo recurrence between groups 1 
and 2. This was demonstrated by a binary logistic regression that produced an odds ratio of vertigo recurrence in group 1 patients as compared with group 2 patients of 1.49 , but with a $95 \%$ CI of 0.37-5.9.

Patients with no vertigo recurrence had a mean posttreatment AVOR gain of $0.46 \pm 0.21$ (range 0.23-1.05, median 0.38), while patients who suffered from recurrent vertigo had a mean post-treatment AVOR gain of $0.57 \pm$ 0.25 (range $0.18-1.02$, median 0.60 ). This trend towards lower AVOR gain in patients who did not experience recurrence did not quite reach statistical significance $(\mathrm{p}=$ 0.09, Mann-Whitney U test, power $=75.7 \%$ ). Additionally, no significant correlation was found between the post-treatment time to recurrence of vertigo and the ipsilateral horizontal canal gain following the first gentamicin injection (graph not shown).

No correlation was found between continuous variables of vertigo rate and AVOR gain following the first IT gentamicin injection $\left(r^{2}=0.01\right.$ with and without outliers, graph not shown).

Three patients continued to experience a high number of monthly vertigo episodes, approximately 8-25 attacks per month, following the first IT gentamicin injection. However, the severity of the attacks was diminished in these cases. The first patient (8 episodes/month) had suffered severe hours-long episodes of spinning vertigo prior to the first treatment, but after this treatment he experienced only mild disequilibrium and vertigo attacks. The second patient (also 8 episodes/month) had had disabling attacks of vertigo on a daily basis prior to the first treatment, with attacks often lasting approximately $2 \mathrm{~h}$ and followed by prolonged equilibrium. After the first treatment, she reported some minor and major attacks of vertigo. The third patient (25 episodes/month) did not notice a change in the frequency of attacks following her first treatment, but did report that the episodes of vertigo were much less severe than before.

No correlation was found between the percentage of original vertigo rate remaining after the first IT gentamicin treatment and the post-treatment ipsilateral horizontal canal AVOR gain $\left(\mathrm{r}^{2}=0.01\right.$ with and without outliers, graph not shown).

\section{Later Gain Changes}

In figure 2, the data for patient $\mathrm{X}$ demonstrate what might be expected for the AVOR gain: a decrease after IT gentamicin treatment concomitant with good vertigo control, but then an increase corresponding to recurrence of vertigo. Before IT gentamicin treatment (fig. 2a), this patient suffered daily attacks of vertigo, and his ipsi-

Vestibular Function and Vertigo Control after IT Gentamicin for Ménière’s Disease lateral horizontal canal AVOR gain was measured to be 0.91 . One year after treatment, his AVOR gain had fallen to 0.18 , and he had complete vertigo control (fig. $2 b$ ). However, 2 years after treatment, he suffered recurrence of several episodes of vertigo, and at this time his AVOR gain had risen to 0.44 (fig. 2c). In this case, vertigo recurrence paralleled the recovery of AVOR gain, suggesting that a return of function in his horizontal semicircular canal contributed to recurrence of vertigo.

Patient $Y$ also had a fairly normal pre-treatment AVOR gain of 0.77 and suffered daily attacks of vertigo before treatment (fig. 2d). Two years after treatment, he experienced complete vertigo control in conjunction with a reduced AVOR gain of 0.51 (fig. 2e). One year later, he began to suffer recurrence of vertigo at a rate of approximately 1 episode per day. At this point in time, one might expect to observe a recovery of AVOR gain corresponding with vertigo recurrence. However, the unexpected finding was that the AVOR gain remained at its initial post-treatment value of 0.51 (fig. $2 \mathrm{f}$ ). Thus, in this case it would seem that vertigo recurrence did not correspond to recovery of AVOR gain measured with the HTT.

Out of 23 patients for whom both early and late posttreatment data are available, 8 patients had post-treatment changes in vertigo frequency that paralleled the changes in AVOR gain as exemplified by patient X. For 4 of these patients, there was a decrease in AVOR gain immediately after IT gentamicin treatment, accompanied by a decrease in vertigo rate. Subsequently, AVOR gain remained stable at the reduced value in these 4 , and vertigo rates did not increase. For 2 patients, AVOR gain decreased immediately after IT gentamicin treatment and decreased further at the second measurement. Vertigo rates decreased and remained decreased in these 2 cases. For 2 patients, however, AVOR gain and vertigo rates decreased immediately after IT gentamicin treatment, but AVOR gain subsequently increased in concurrence with increases in vertigo rates.

The remaining 15 patients did not show direct correlations between post-treatment changes in vertigo frequency and AVOR gain, similar to patient Y. For example, in 5 patients, AVOR gain decreased immediately after IT gentamicin treatment, but subsequent increases in AVOR gain were not accompanied by increases in vertigo rates. In 2 patients, vertigo rates increased despite a stable reduction in AVOR gain over time after IT gentamicin treatment.

Table 2 is a contingency table that was constructed for 17 patients, each of whom had an immediate decrease in AVOR gain following IT gentamicin treatment. AVOR 


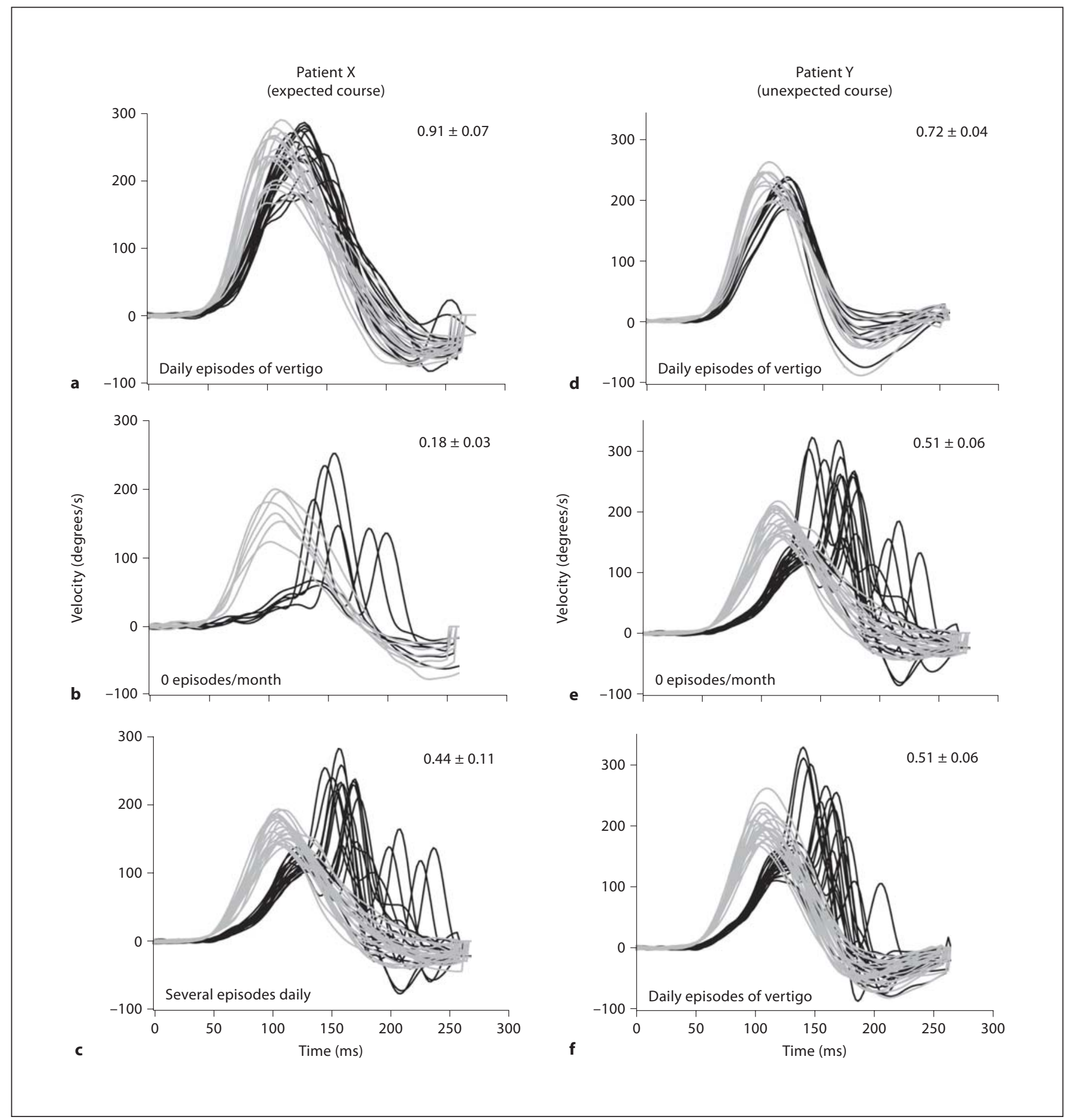

Fig. 2. Head and eye velocity traces in response to passive head movements in the plane of the horizontal semicircular canal for 2 patients before and after gentamicin treatment, patient $X(\mathbf{a}-\mathbf{c})$ and patient $Y(\mathbf{d}-\mathbf{f})$. Pre-gentamicin traces $(\mathbf{a}, \mathbf{d})$, early post-gentamicin traces $(\mathbf{b}, \mathbf{e})$, and late post-gentamicin traces $(\mathbf{c}, \mathbf{f})$ are shown. Gray traces represent head velocity and black traces represent the corresponding eye velocity; horizontal canal AVOR gain value was calculated as the ratio of eye velocity to head veloc- ity and is noted in the upper right corner. Vertigo rate is noted in the lower left corner of each panel. Note that sudden increases in eye velocity occurring $>70 \mathrm{~ms}$ after onset of head movement correspond to refixation saccades. The gain calculations were done on data prior to the appearance of these refixation saccades to ensure that only vestibularly-driven eye movements were considered. 
gain values and vertigo rates in the table correspond to later post-treatment measurements. Of the 6 patients who had increases in gain, only 1 had a corresponding increase in vertigo rate. Of the 11 patients who had stable decreases in gain, 2 had increases in vertigo rate. Fisher's exact probability test yielded a two-tailed p value of 1.000, indicating that there was no significant difference in the direction of change of the vertigo rate between patients who had an increase in AVOR gain at a late post-treatment time point versus those that had a decrease or no change in gain.

Data from 3 or more sessions of quantitative head thrust testing were available for a subset of 22 patients, 11 of whom did not have recurrent vertigo, and 11 of whom did. These data were analyzed to determine the percentage recovery in AVOR gain that occurred between the second test (immediately after gentamicin) and the third test (long-term follow-up, 129-1299 days). Patients who did not experience recurrent vertigo had a mean percent recovery in AVOR gain of $11 \pm 16 \%$, while those who did suffer recurrence had a mean percent recovery of $-1.97 \pm 24.93 \%$. This difference was not statistically significant (Mann-Whitney $U$ analysis, $\mathrm{p}=$ $0.19)$.

\section{Section IV - Caloric Results}

Figure 3 displays post-treatment unilateral weakness (UW) after the first IT gentamicin injection in patients who achieved sufficient vertigo control after a single injection and in those who required multiple injections. Patients in the single treatment group $(\mathrm{n}=16)$ had a mean post-treatment UW after the first treatment of $66.9 \pm$ $31.8 \%$ (range $4-100 \%$, median $68.5 \%$ ), while patients in the multiple treatment group $(\mathrm{n}=20)$ had a mean posttreatment UW of $38.9 \pm 30.4 \%$ (range 1-100\%, median 38.5\%). A Mann-Whitney $U$ analysis showed that there was a statistically significant difference between these 2 groups $(\mathrm{p}=0.0137)$.

No significant correlation was observed between time to recurrence and UW (graph not shown). Most recurrences of vertigo were found to occur within the first 200 days after treatment.

Table 3 shows the probability that a patient needed a repeat IT gentamicin injection, based on his or her caloric UW. Patients with a UW of less than or equal to $50 \%$ had a $79 \%$ chance of needing a repeat IT gentamicin injection, whereas patients with a UW of greater than $50 \%$ had a $31 \%$ chance of needing another injection. A binary logistic regression produced an odds ratio of needing a repeat injection of $0.12(95 \%$ CI $0.03-0.56)$ in

Vestibular Function and Vertigo Control after IT Gentamicin for Ménière’s Disease

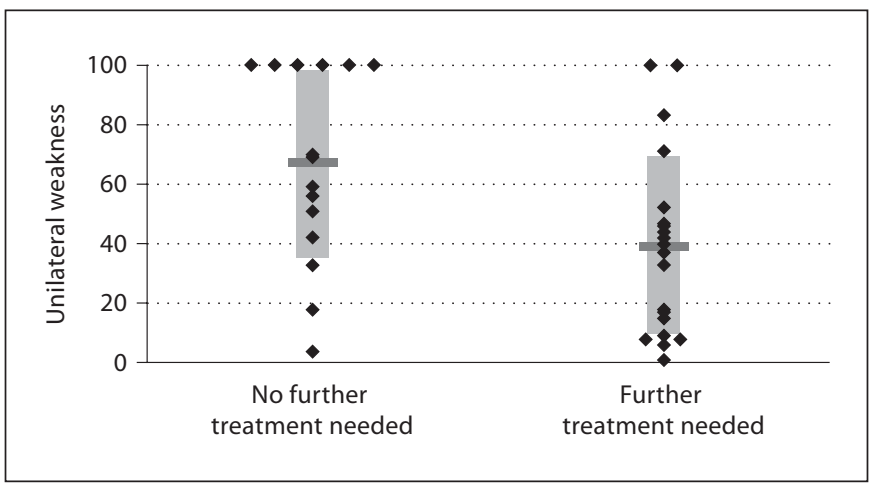

Fig. 3. Caloric UW after first IT gentamicin injection in patients who received single or multiple rounds of treatment. Diamonds represent single patients, and horizontal bars denote the mean for each group.

Table 2. Contingency table for 17 patients who had an initial decrease in gain at the early post-treatment time point

\begin{tabular}{lllr}
\hline & \multicolumn{2}{l}{ AVOR gain } \\
\cline { 2 - 4 } & increase & $\begin{array}{l}\text { decrease or } \\
\text { no change }\end{array}$ & total \\
& & & \\
\hline $\begin{array}{l}\text { Vertigo rate } \\
\text { Increase }\end{array}$ & 1 & 2 & 3 \\
Decrease or no change & 5 & 9 & 14 \\
Total & 6 & 11 & 17 \\
\hline
\end{tabular}

Gain values and vertigo rates correspond to the late post-treatment time points. Fourteen patients had a stable decrease in vertigo rate, while 3 patients had an increase in vertigo rate at this late post-treatment time point. One of these 3 patients had complete vertigo control immediately following IT gentamicin treatment, but reported 1 episode of vertigo at a later follow-up time point, and 2 patients had changes of 0 episodes/month increasing to 6 episodes/month, and 0 episodes/month increasing to 0.3 episodes/month. AVOR gain increased for 6 patients (by $41.48 \% \pm$ 9.59\%) between the early and late post-treatment time points, but decreased (by $25.88 \% \pm 9.31 \%$ ) for 3 patients or did not change for 8 patients (11 total patients).

Table 3. Probability of needing a repeat injection

Patients needing Patients not needing repeat injection repeat injection

\begin{tabular}{rrr}
\hline $\mathrm{UW} \leq 50 \%$ & $15(79)$ & $4(21)$ \\
$\mathrm{UW}>50 \%$ & $5(31)$ & $11(69)$
\end{tabular}

Figures in parentheses are percentages. 




Fig. 4. Post-treatment AVOR gain versus post-treatment caloric UW. Linear best-fit line is shown.

patients with a UW $>50 \%$ as compared with patients with a UW $\leq 50 \%$. Thus, those patients with $>50 \%$ caloric UW after one IT gentamicin injection were less likely to need a repeat injection than those with $\leq 50 \%$ UW.

\section{Section V-Post-Treatment AVOR Gain and Caloric $U W$}

Figure 4 depicts post-first-treatment ipsilateral horizontal canal AVOR gain versus caloric UW for each of 36 patients. There was a weak correlation between decreasing AVOR gain and increasing UW (linear regression, $\mathrm{R}^{2}=0.26$ ). While there was a large spread of AVOR gain values for most values of caloric UW, the spread was narrower for patients with complete UW (AVOR gain range $0.24-0.46)$.

Only 2/10 (20\%) of patients from figure 4 with a posttreatment $\mathrm{UW}<20 \%$ had a post-treatment AVOR gain of $\leq 0.5$, whereas all $8 / 8(100 \%)$ of patients with a post-treatment UW of $100 \%$ had a post-treatment AVOR gain of $\leq 0.5$. Fisher's exact test demonstrated that patients with UW of $100 \%$ were significantly more likely to have an AVOR gain below 0.5 than patients with UW $<20 \%$ (2tailed $\mathrm{p}$ value $<0.05$ ).

Of the 8 patients who had complete unilateral caloric weakness, post-first-treatment clinical HTT results were available for 7 patients. Three of these patients had markedly diminished, 3 had diminished, and 1 had mildly diminished HTT results.

\section{Discussion}

AVOR Gain Reduction, Caloric Reduction, and Vertigo Control

The aim of this study was to examine long-term vertigo control and vertigo recurrence in patients with unilateral Ménière's disease treated with IT gentamicin, and to examine relationships between control of vertigo and measures of peripheral vestibular function, including the qualitative clinical head thrust sign, quantitative horizontal semicircular canal AVOR gain, and caloric weakness. We found that the best predictors of vertigo control appeared to be: (1) reduction in quantitative horizontal semicircular canal AVOR gain to below $40 \%$ of the original gain, and/or (2) caloric UW of greater than $50 \%$.

The present study confirms that greater reductions in AVOR gain correspond to lower rates of vertigo after IT gentamicin treatment. The data in table 1 demonstrate that IT gentamicin treatment is often highly effective at reducing the rate of vertigo, no matter what degree of functional deficit is reached in the labyrinth. However, if vertigo recurs, the frequency of vertigo attacks at the time of recurrence is significantly less if the AVOR gain has been reduced by the first IT gentamicin treatment to below $40 \%$ of the starting value than if it remains $\geq 40 \%$.

Patients commonly chose to have repeated IT gentamicin injections for recurrent vertigo even when vertigo occurred at much reduced rates in comparison to their starting rates. In fact, the $95 \%$ CI for the odds ratio of some recurrence of vertigo comparing groups with $<40 \%$ or $\geq 40 \%$ reduction in AVOR gain spanned the number one. Thus, greater reductions in AVOR gain may not necessarily obviate requests for repeated IT gentamicin injections. This reflects the unique nature of IT gentamicin titration. Once patients are aware that an office treatment might relieve vertigo, the level of vertigo tolerated may decrease with subsequent recurrences. Thus, recurrent vertigo treated with IT gentamicin does not necessarily reflect a return to the same vertigo burden suffered before IT gentamicin treatment.

The distinction between objective vertigo reduction and the patient's subjective and changing perception of adequate vertigo control may explain why the findings of this study appear to differ from those previously documented by our laboratory. Lin et al. [2005] found that there was a significantly greater change in ipsilateral horizontal canal gain in patients who achieved complete vertigo control with only one IT gentamicin injection versus those who required multiple injections, suggesting that there was an association between horizontal canal func- 
tion and vertigo control. With the present study, it is clear that patients may choose to repeat IT gentamicin injections for lower levels of vertigo at the time of recurrence. Thus, greater AVOR gain reduction may predict lower vertigo rates, but not necessarily lower rates of retreatment with IT gentamicin. Furthermore, the differences in this and the previous study might be related to followup time. That study was limited to the vertigo control obtained in the first year following initiation of IT gentamicin treatment. Figure 1 in this study demonstrates that vertigo may recur beyond a year after IT gentamicin treatment. The inclusion of vertigo occurring after 1 year may explain the lack of correlation between AVOR gain and vertigo control when these values were treated as continuous variables.

There were some other surprising negative findings in this study. For example, we did not observe any association between vertigo rate and AVOR gain when these were treated as continuous variables. We also did not find any correlation between AVOR gain and caloric UW. Finally, the clinical head thrust sign did not prove to be a successful predictor of vertigo control. There are a number of factors that may explain these negative findings.

First of all, the well-known high spontaneous rate of vertigo remission in Ménière's disease undoubtedly confounds the findings of this study. Silverstein et al. [1989] followed 50 surgical candidates who declined surgical treatment for Ménière's disease, and found that after 2 years of follow-up, 57\% had complete vertigo control, and after an average of 8.7 years, $71 \%$ had achieved complete control. It is likely that the spontaneous remission rate continues to be substantial after IT gentamicin treatment, and such cases of remission may mask any correlations between continuous variables of gain reduction and vertigo control.

Secondly, the HTT has unique features that should be considered in interpreting the results. The test uses a high-frequency, high-velocity, high-acceleration head turn that delivers a robust stimulus to the horizontal semicircular canal. In contrast, the caloric stimulus engenders relatively modest convective endolymph flow at very low frequencies. The intense nature of the head thrust stimulus may overcome partial weakness in the semicircular canal unless the canal deficit is especially severe. As such, the HTT may yield very different results than the caloric test. In its qualitative version, the HTT has even more limitations. A 'positive' HTT depends upon the appearance of a refixation saccade after the head has stopped moving or is close to its halt. Recent studies have demonstrated that this finding depends

Vestibular Function and Vertigo Control after IT Gentamicin for Ménière’s Disease upon examiner experience, and that experienced examiners are, in fact, less likely to declare a positive sign (refixation saccade) than novice examiners [Jorns-Haderli et al., 2007]. However, when detected, the head thrust sign is a highly specific test of whether or not there has been an ablative lesion on the labyrinth [Minor, 1999; Melvin et al., 2009].

Thirdly, the tests used in this study are limited to those of semicircular canal function. It is possible that canal tests such as the HTT and caloric test may not be effective predictors of vertigo control if, in addition to the semicircular canals, the otolith organs also play a role in labyrinthine dysfunction. Indeed, histopathological studies of temporal bones from individuals with Ménière's disease have demonstrated severe hydrops in the saccule, utricle, cochlea, and semicircular canals [Fraysse et al., 1980; Okuno and Sando, 1987; Rauch et al., 1989]. Additionally, VEMP test results, which are indicative of saccular function, have been found to be abnormal in many patients with Ménière's disease, and especially those with Ménière's disease who have a history of drop attacks [de Waele et al., 1999; Timmer et al., 2006].

Both animal and human studies have reported damage to the saccule and utricle following IT gentamicin treatment. VEMPs have been found to be absent from $100 \%$ of guinea pig ears and human ears after treatment with gentamicin [Day et al., 2007b]. Subjective visual vertical test responses, which evaluate utricular function, have been found to be abnormal in human patients in the early period following treatment [Helling et al., 2007]. Histopathological analyses have demonstrated atrophy and distortion of the sensory cells of the saccular macula in the guinea pig, and severe type I and type II vestibular hair cell loss in the utricular macula of humans following gentamicin treatment [Day et al., 2007; Ishiyama et al., 2007].

However, although IT gentamicin appears to affect the otolith organs in addition to the semicircular canals, several studies that have examined the differential sensitivity of the vestibular end organs to gentamicin and streptomycin have demonstrated that these drugs exhibit milder toxicity to the otolith organs than to the semicircular canals. IT administration of streptomycin to the guinea pig caused the greatest degeneration in the sensory epithelia of the crista ampullaris, followed by lesser degeneration in the utricular macula, and even milder degeneration in the saccular macula [Lindeman, 1969]. Similarly, the sensory epithelium of the chicken utricle was shown to suffer less damage than that of the horizontal canal cristae ampullaris after intramuscular strepto- 
mycin injection [Weisleder and Rubel, 1993]. Moreover, Hirvonen et al. [2005] found that there was a 90\% reduction in semicircular canal afferent rotational sensitivity, but only a $77 \%$ reduction in utricular canal afferent tilt sensitivity, in chinchillas treated with gentamicin.

Such differential vulnerability to aminoglycoside antibiotics suggests relative sparing of the otolith organs. Thus, recurrent vertigo could be mediated principally by the utricle and saccule. If this were true, it might explain why we did not find closer correlations between vertigo rates and measures of semicircular canal function.

Conversely, vertigo control may not be related predominantly to hair cell mechanisms like transduction or synaptic activity, but may instead depend on the firing of vestibular afferents. Perhaps vertigo occurs as the result of an underlying disorder affecting vestibular nerve afferent firing rates, independent of hair cell input. In this case, vertigo recurrence would be unaffected by any measure of canal sensory transduction. In this regard, it is notable that a histopathological study demonstrated loss of ganglion cells as well as type II hair cells in vestibular tissues from humans with Ménière's disease [Tsuji et al., 2000]. Furthermore, data from patients with Ménière's disease who have received cochlear implants suggest a possible fluctuation in afferent function: some of these patients report fluctuations in hearing in the implanted ear in association with fluctuations in aural fullness [Lustig et al., 2003].

\section{Difficulty in Estimating Post-Treatment Vertigo \\ Frequency}

Vertigo rates were calculated from questionnaires asking patients to provide the number of attacks of vertigo they had suffered since their last clinic visit (estimating vertigo frequency). Vertigo rates were calculated from questionnaires asking patients to provide the number of attacks of vertigo they had suffered since their last clinic visit; therefore, these data were subject to recall bias. A more accurate method would have been to utilize daily patient logs to record vertigo attacks. However, even a prospectively designed and measured estimate of vertigo frequency would still be highly subject to variability in any protocol in which patients are given the option of repeating gentamicin injections 'as needed'. The expectation of a treatment that can be easily repeated when vertigo frequency and/or severity are increasing will inevitably lead to requests for such treatment shortly into the course of worsening vertigo. In this situation, getting an accurate monthly estimate of vertigo frequency is difficult. Such an estimate may be based on only 1 or 2 ver- tigo spells. A relatively short time separating 2 spells (the denominator in the rate calculation) can drastically affect the estimate of vertigo frequency.

Additionally, another limitation of our analysis was our failure to classify and discriminate vertigo attacks based on severity. Clinically, it was noted that patients often experienced milder episodes of vertigo following IT gentamicin treatment, although the true number of attacks may not have fallen. Examination of individual records belonging to several patients who were noted to have increased vertigo rates after treatment showed that these individuals did report milder vertigo attacks and less disequilibrium after treatment. IT gentamicin may thus be more effective than our rate-based analysis would suggest. A more comprehensive analysis would have examined both the number and severity of vertigo episodes before and after treatment.

\section{Caloric Testing}

We found that patients with a post-treatment caloric UW of greater than $50 \%$ had a significantly lower chance of needing a repeat gentamicin injection than patients who had a UW of $\leq 50 \%$. In addition, patients with a post-treatment UW of $100 \%$ were significantly more likely to have a post-treatment AVOR gain of $\leq 0.5$ than patients with a post-treatment UW of $\leq 20 \%$. Patients who achieved sufficient vertigo control after a single injection had a significantly greater value of post-first-treatment UW than those who required multiple injections. However, considering all patients, the difference in mean UW between patients who were vertigo-free and those who experienced recurrent vertigo did not quite reach statistical significance.

The lack of association between vertigo recurrence and UW was not entirely unexpected, given our negative findings regarding the association between vertigo rate and AVOR gain when these were treated as continuous variables. It is likely that both of these negative findings may be attributed to the same factors, namely the patient's changing level of tolerance of vertigo attacks, and his or her subjective perception of the satisfactory level of vertigo control.

Perhaps due to this and other factors, previous investigations have reported variable findings in association with the caloric test. Lin et al. [2005] reported that singletreatment patients had a pre-treatment to post-treatment change in caloric asymmetry of $25.3 \pm 30.0$, while multiple-treatment patients had a similar change of $23.8 \pm$ 31.3. Chung et al. [2007] found significantly decreased caloric responses in $85 \%$ of single-treatment patients and $88 \%$ of multiple-treatment patients; caloric responses 
were absent in $23 \%$ of single-treatment patients and $37 \%$ of multiple-treatment patients. Other studies have also shown that there are not significant associations between post-treatment AVOR gain and post-treatment caloric weakness [Carey et al., 2002; Lin et al., 2005; Helling et al., 2007]. Carey et al. [2002] showed that subjects with absent caloric responses following IT gentamicin treatment had a horizontal canal gain of $0.40 \pm 0.11$, while subjects who retained their caloric responses had a horizontal canal gain of $0.41 \pm 0.17$.

\section{Conclusion}

Satisfactory control of vertigo following a single IT gentamicin injection was associated with a reduction in quantitative ipsilateral horizontal semicircular canal AVOR gain to below $40 \%$ of the original value and ca- loric UW greater than $50 \%$ after that injection. However, no significant correlations were found between vertigo control and either gain or gain recovery when these were treated as continuous variables, nor between gain and caloric UW. There were no significant differences in gain, gain recovery, or caloric UW between patients who did and did not have some recurrence of vertigo. Patients may choose to receive repeated IT gentamicin injections for recurrent vertigo that is less frequent or severe than what they originally experienced.

\section{Acknowledgments}

This study was supported by NIH R01 DC05040 (J.P.C.) and by a grant from the Doris Duke Charitable Foundation to the Johns Hopkins University School of Medicine to fund a Clinical Research Fellow (K.D.N.). We would like to thank Gautam Bhatia, Seun Myrie, and Steven Sweet for assistance in data analysis.

\section{References}

Bodmer D, Morong S, Stewart C, Alexander A, de Waele C, Meguenni R, Freyss G, Zamith F, Chen JM, Nedzelski JM: Long-term vertigo control in patients after intratympanic gentamicin instillation for Ménière's disease. Otol Neurotol 2007;28:1140-1144.

-Carey JP, Minor LB, Peng GC, Della Santina CC, Cremer PD, Haslwanter T: Changes in the three-dimensional angular vestibulo-ocular reflex following intratympanic gentamicin for Meniere's disease. J Assoc Res Otolaryngol 2002;3:430-443.

-Chia SH, Gamst AC, Anderson JP, Harris JP Intratympanic gentamicin therapy for $\mathrm{Me}$ niere's disease: a meta-analysis. Otol Neurotol 2004;25:544-552.

Chung WH, Chung KW, Kim JH, Cho YS, Hong $\mathrm{SH}$ : Effects of a single intratympanic gentamicin injection on Meniere's disease. Acta Otolaryngol S 2007;558:61-66.

Cohen-Kerem R, Kisilevsky V, Einarson TR, Kozer E, Koren G, Rutka JA: Intratympanic gentamicin for Meniere's disease: a metaanalysis. Laryngoscope 2004; 114:20852091.

da Costa S, de Sousa LC, Piza MR: Meniere's disease: overview, epidemiology, and natural history. Otolaryngol Clin North Am 2002; 35:455-495.

Day AS, Lue JH, Yang TH, Young YH: Effect of intratympanic application of aminoglycosides on click-evoked myogenic potentials in guinea pigs. Ear Hear 2007;28:18-25.

de Waele C, Huy PT, Diard JP, Freyss G, Vidal PP: Saccular dysfunction in Meniere's disease. Am J Otol 1999;20:223-232.

Vestibular Function and Vertigo Control after IT Gentamicin for Ménière’s Disease Bellalimat N, Vidal PP, Huy PTB: Intratympanic gentamicin injections for Meniere disease - vestibular hair cell impairment and regeneration. Neurology 2002;59:14421444.

Fraysse B, Alonso A, House WF: Meniere's disease and endolymphatic hydrops: clinicalhistopathological correlations. Ann Otol Rhinol Laryngol Suppl 1980;89:2-22.

-Helling K, Schonfeld U, Clarke AH: Treatment of Meniere's disease by low-dosage intratympanic gentamicin application: effect on otolith function. Laryngoscope 2007;117:22442250.

Hirvonen TP, Minor LB, Hullar TE, Carey JP: Effects of intratympanic gentamicin on vestibular afferents and hair cells in the chinchilla. J Neurophysiol 2005;93:643-655.

Hone SW, Nedzelski J, Chen J: Does intratympanic gentamicin treatment for Meniere's disease cause complete vestibular ablation? J Otolaryngol 2000;29:83-87.

- Ishiyama G, Lopez I, Baloh RW, Ishiyama A: Histopathology of the vestibular end organs after intratympanic gentamicin failure for Meniere's disease. Acta Otolaryngol 2007; 127:34-40.

Jongkees LBW, Maas JPM, Philipszoon AJ: Clinical nystagmography: a detailed study of electronystagmography in 341 patients with vertigo. Pract Otorhinolaryngol (Basel) 1962;24:65-93.

Jorns-Haderli M, Straumann D, Palla A: Accuracy of the bedside head impulse test in detecting vestibular hypofunction. J Neurol Neurosurg Psychiatry 2007;78:1113-1118.
Kotimaki J, Sorri M, Aantaa E, Nuutinen J: Prevalence of Meniere disease in Finland. Laryngoscope 1999;109:748-753.

Lange G: Gentamicin and other ototoxic antibiotics for the transtympanic treatment of Meniere's disease. Arch Otorhinolaryngol 1989; 246:269-270.

- Lin FR, Migliaccio AA, Haslwanter T, Minor LB, Carey JP: Angular vestibulo-ocular reflex gains correlate with vertigo control after intratympanic gentamicin treatment for $\mathrm{Me}$ niere's disease. Ann Otol Rhinol Laryngol 2005; 114:777-785.

- Lindeman HH: Regional differences in sensitivity of the vestibular sensory epithelia to ototoxic antibiotics. Acta Otolaryngol (Stockh) 1969;67:177-189.

Lustig LR, Yeagle J, Niparko JK, Minor LB: Cochlear implantation in patients with bilateral Meniere's syndrome. Otol Neurotol 2003;24: 397-403.

-Melvin TA, Della Santina CC, Carey JP, Migliaccio AA: The effects of cochlear implantation on vestibular function. Otol Neurotol 2009; 30:87-94.

Minor LB: Intratympanic gentamicin for control of vertigo in Meniere's disease: vestibular signs that specify completion of therapy. Am J Otol 1999;20:209-219.

Minor LB, Goldberg JM: Influence of static head position on the horizontal nystagmus evoked by caloric, rotational and optokinetic stimulation in the squirrel monkey. Exp Brain Res 1990;82:1-13. 
Monsell EM, Balkany TA, Gates GA, Goldenberg RA, Meyerhoff WL, House JW: Committee on Hearing and Equilibrium guidelines for the diagnosis and evaluation of therapy in Meniere's disease. Otalaryngol Head Neck Surg 1995;113:181-185.

Nguyen KD, Minor LB, Della Santina CC, Carey JP: Time course of repeated intratympanic gentamicin for Meniere's disease. Laryngoscope 2009;119:792-798.

Okuno T, Sando I: Localization, frequency, and severity of endolymphatic hydrops and the pathology of the labyrinthine membrane in Meniere's disease. Ann Otol Rhinol Laryngol 1987;94:438-445.

Paige GD: Caloric responses after horizontal canal inactivation. Acta Otolaryngol (Stockh) 1985; 100:321-327.

- Proctor L, Dix R, Hughes D, Rentea R: Stimulation of the vestibular receptor by means of step temperature changes during continuous aural irrigation. Acta Otolaryngol (Stockh) 1975;79:425-435.
Rauch SD, Merchant SN, Thedinger BA: Meniere's syndrome and endolymphatic hydrops: double-blind temporal bone study. Ann Otol Rhinol Laryngol 1989;98:873883.

Silverstein H, Smouha E, Jones R: Natural history vs. surgery for Meniere's disease. Otalaryngol Head Neck Surg 1989;100:616.

Stahle J, Stahle C, Arenberg K: Incidence of Meniere's disease. Arch Otolaryngol Head Neck Surg 1978;104:99-102.

Straumann D, Zee DS, Solomon D, Lasker AG, Roberts DC: Transient torsion during and after saccades. Vis Res 1995;35:3321-3334.

Tabak S, Collewijn H, Boumans LJJM, Van der Steen J: Gain and delay of human vestibuloocular reflexes to oscillation and steps of the head by a reactive torque helmet. I. Normal subjects. Acta Otolaryngol (Stockh) 1997; 117:785-795.

Tian J, Crane BT, Demer JL: Vestibular catch-up saccades in labyrinthine deficiency. Exp Brain Res 2000;131:448-457.
Timmer F, Zhou G, Guinan JJ, Kujawa SG, Hermann B, Rauch SD: Vestibular evoked myogenic potential (VEMP) in patients with Meniere's disease with drop attacks. Laryngoscope 2006;116:776-779.

Tsuji K, Velazquez-Villasenor L, Rauch SD, Glynn RJ, Wall C 3rd, Merchant SN: Temporal bone studies of the human peripheral vestibular system: Meniere's disease. Ann Otol Rhinol Laryngol Suppl 2000;181:26-31.

-Watanabe Y, Mizukoshi K, Shojaku H, Watanabe I, Hinoki M, Kitahara M: Epidemiological and clinical characteristics of Meniere's disease in Japan. Acta Otolaryngol Suppl (Stockh) 1995;519:206-210.

Weisleder P, Rubel EW: Hair cell regeneration after streptomycin toxicity in the avian vestibular epithelium. J Comp Neurol 1993;331: 97-110.

Wladislavosky-Waserman P, Facer GW, Bahram M, Kurland LT: Meniere's disease: a 30-year epidemiologic and clinical study in Rochester, MN. 1951-1980. Laryngoscope 1984;94: 1098-1102. 